\title{
FINITE PRANDTL NUMBER CONVECTION WITH NEARLY INSULATING BOUNDARIES
}

\author{
N. RIAHI
}

\begin{abstract}
Arbitrary Prandtl number convection in a layer with nearly insulating boundaries is investigated. For $B^{1 / 3}>3.95 P$ ( $P$ is the Prandtl number and $B$ being the ratio between the thermal conductivities of the boundary and of the fluid) two-dimensional rolls are stable. The heat transported by the stable rolls reaches its peak at a critical $P=B^{1 / 3} / 5.77$ beyond which the heat flux decreases with increasing $P$. For $B^{1 / 3}=3.95 P$ rolls become unstable to disturbances in the form of rolls oriented at a right angle to the original rolls. For $B^{1 / 3}<3.95 P$ square pattern convection represents the preferred stable convection. The stable square pattern transports the maximum amount of heat at a critical $P=B^{1 / 3} / 3.7$.
\end{abstract}

\section{Introduction}

In two recent studies of finite amplitude convection in a layer with nearly insulating boundaries Busse and Riahi [3] and Riahi [4], it was found that for the Prandtl number $P$ vanishingly small two-dimensional rolls are the preferred stable convection pattern, while square pattern convection represents the preferred stable convection for infinitely large $P$. The present study is concerned with the arbitrary Prandtl number convection case and includes a detailed analysis of the transition range

Received 28 May 1981. 
from rolls to square cells and vice versa. An important result of the present study and in sharp contrast to the corresponding problem with isothermal boundaries (Schlüter et al [5]) is that the heat transported by the preferred stable flow pattern exhibits a non-monotonicity behavior with respect to $P$ at a given Rayleigh number $R$ in a low Prandtl number fluid. The background, motivation and derivation of the governing equations for the problem can be found in [3] and [4]. Consequently the governing equations are derived briefly in Section II and only the essential details of the finite amplitude convection are given and discuss discussed in Section III.

\section{Governing equations}

We consider an infinite horizontal layer of fluid of depth $d$ bounded by two infinite half spaces with the thermal conductivity $\lambda^{e}$ which is assumed to be small compared to the thermal conductivity $\lambda$ of the fluid. In the steady static state, a constant heat flux traverses the system such that the temperatures $T_{1}$ and $T_{2}$ are attained at the upper and lower boundaries of the fluid. Under the usual Boussinesqr approximation, the non-dimensional forms of the equations for momentum, heat and conservation of mass can be simplified by using the general representation for the velocity vector as the sum of the poloidal $\nabla x \nabla x \lambda v$ and toroidal $\nabla x \lambda \psi$ vectors where $\lambda$ represents a unit vector in vertical direction. The detailed analysis, though omitted here, indicates that the terms containing $\psi$ in the basic equations are essentially insignificant, since the toroidal component of the velocity vector is of the order of the $m$ th power of the amplitude $\varepsilon \quad(m \geq 3)$ and thus cannot enter the small amplitude analysis discussed in this paper. Therefore, we simply set $\psi=0$ in the basic equations for the subsequent analysis to be discussed in the next section. Vertical component of the double curl of the momentum equation together with the heat equation then yield the following governing equations

$$
\begin{gathered}
\nabla^{4} \Delta_{2} v-\Delta_{2} \theta-\frac{\partial}{\partial t} \nabla^{2} \Delta_{2} v=\delta \cdot(\delta v \cdot \nabla \delta v), \\
\nabla^{2} \theta-R \Delta_{2} v-P \frac{\partial \theta}{\partial t}=P \delta v \cdot \nabla \theta,
\end{gathered}
$$

where $\delta=\nabla x \nabla x \lambda, \theta$ is the deviation of the temperature from its static 
value, $R=\alpha\left(T_{2}-T_{1}\right) g d^{3} \rho_{0} c / \nu \lambda$ is the Rayleigh number, $p=\nu \rho_{0} c \lambda^{-1}$ is the Prandt 1 number, $\rho_{0}$ is the reference density, $c$ is the specific heat at constant pressure, $\alpha$ is the coefficient of thermal expansion, $v$ is the kinematic viscosity, $g$ is acceleration due to gravity and $\Delta_{2}$ is the horizontal Laplacian.

Following [3], the boundary conditions for $v$ and $\theta$ are

$$
v=\frac{\partial v}{\partial z}=\frac{\partial \theta}{\partial z} \pm a B \theta=0 \text { at } z= \pm \frac{1}{2},
$$

where $a$ is the horizontal mere number of the linear planform function $w(x, y)=\sum_{n=-N}^{N} c_{n} \exp \left(i k_{n} \cdot \Gamma\right), B=\lambda^{e} / \lambda, r$ is the position vector and the horizontal wave number vectors $k_{n}$ satisfy the properties

$$
\mathrm{k}_{n} \cdot \lambda=0, \quad\left|\mathrm{k}_{n}\right|=a, \mathrm{k}_{-n}=-\mathrm{k}_{n} .
$$

The coefficients $c_{n}$ satisfy the conditions

$$
\sum_{n=-N}^{N} c_{n} c_{n}^{*}=1, \quad c_{n}^{*}=c_{-n}
$$

where $c_{n}^{*}$ denotes the complex conjugate of $c_{n}$. The linear planform function $w$ and (3), (4) are now standard in thermal convection theory. The reader is referred to the recent review of the problem of thermal convection, Busse [2], for details on the subject.

\section{Finite amplitude convective motion}

Since the linear problem of the present study is identical to that discussed in [3], it is found again that the value $a_{c}$ which minimizes $R$ is proportional to $B^{1 / 3}$. Thus it is assumed that $a^{2}=\eta^{2} \gamma$, where $Y \equiv B^{2 / 3}$ and $\eta$ is of the order unity for the convection modes of physical interest. We then seek solutions in terms of a double series in powers of $\gamma$ and $E$ : 


$$
\left(\begin{array}{l}
v \\
\theta
\end{array}\right)=\sum_{n=0, m=1} \varepsilon^{m} \gamma^{n}\left(\begin{array}{c}
v_{m}^{(n)} \\
\theta_{m}^{(n)}
\end{array}\right), \quad R=\sum_{m, n=0} \varepsilon^{m} \gamma^{n} R_{m}^{(n)} .
$$

As we mentioned above, the linear analysis has already been discussed in [3]. It was found in [3] that

$$
\begin{aligned}
\theta_{1}^{(0)}=w(x, y), v_{1}^{(0)}=w(x, y)\left(z^{2}-\frac{1}{4}\right)^{2} / 4 ! & \\
R_{0}^{(0)} & =720, \eta_{c}=(462 / 17)^{1 / 3} .
\end{aligned}
$$

The detailed analysis of the nonlinear problem in [3] and [4] indicates that only the solutions in the orders $\varepsilon^{2} \gamma$ and $\varepsilon^{3} \gamma^{2}$ are needed to form the necessary solvability conditions. It was found in [3] that $\theta_{2}^{(1)}$ was the significant solution which was needed in the nonlinear analysis, while $v_{2}^{(1)}$ and $v_{3}^{(2)}$ were found in [4] to be significant. Keeping the necessary leading order terms in $P$, it is found that $\theta_{2}^{(1)}$ has essentially the same expression as in the case discussed in [4], except a factor of $P$ which is resulted from the present non-dimensionalization procedure. $\theta_{3}^{(2)}$ is found to be insignificant, and the expressions for $v_{2}^{(1)}$ and $v_{3}^{(2)}$ are essentially the same as in the case discussed in [4]. Their expressions are lengthy and are not given here. Since we are interested in discussing the solutions for arbitrary $P$ it is found that the expression

$$
F=\varepsilon^{2} \gamma R_{2}^{(1)}+\varepsilon^{2} \gamma^{2} R_{2}^{(2)}
$$

is turned out to be the largest non-zero term, in the nonlinear domain $(m>1)$, in the expansion (5) for $R$. The expression for $R_{2}^{(1)}$ is found after forming the solvability condition for the heat equation in the order $\varepsilon^{3} \gamma^{2}$. Similarly, $R_{2}^{(2)}$ is found after forming the solvability condition for the heat equation in the order $\varepsilon^{3} \gamma^{3}$. After some considerable algebra, these conditions yield the following system of equations 
(8) $\quad F c_{n}=\frac{n \varepsilon^{2} \gamma^{2}}{2^{15} \cdot 63.221} \sum_{m, l, p}\left(1+\phi_{l p}\right)\left(13884 \phi_{l p}^{2}-24813 \phi_{l p}+76288\right) c_{m} c^{c} l_{p}$

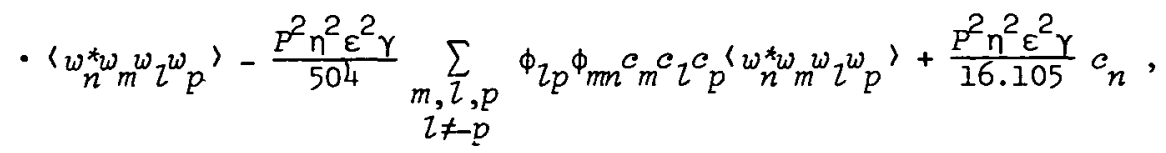

$$
\begin{aligned}
& n=-N, \ldots,-1,1, \ldots, N \text {, }
\end{aligned}
$$

where $\phi_{m n}=\left(k_{m} \cdot k_{n}\right) a^{-2}$ and an angle bracket denotes total horizontal average. The integral expression in (8) differs from zero only if

$$
-k_{n}+k_{m}+k_{2}+k_{p}=0 \text {. }
$$

This condition yields a much simplified set of equations

$$
F c_{n}=\frac{\eta \varepsilon^{2} \gamma}{504} \sum_{m=-N}^{N} T_{n m}\left|c_{m}\right|^{2} c_{n}, n=-N, \ldots,-1,1, \ldots, N \text {, }
$$

where

$$
\text { (10) } T_{n m}=\left\{\begin{array}{l}
\frac{65359 \gamma}{2^{12} \cdot 221}+\frac{4 P^{2} \eta}{5} \text { for } m= \pm n \\
\frac{\gamma}{2^{11} \cdot 221}\left(1+\phi_{m n}\right)\left(13884 \phi_{m n}^{2}-24813 \phi_{m n}+76288\right)+2 P^{2} \eta\left(\phi_{m m}^{2}+\frac{3}{20}\right) \\
\text { otherwise. }
\end{array}\right.
$$

Equations (9) and (4) represent an inhomogeneous system of $2 N+1$ nonlinear algebraic equations for the $2 N$ coefficients $c_{n}$ and the expression $F$. The general solution of this system is not known, but a simple set of solutions can be easily derived in the regular case, in which all angles between two neighboring k-vectors are equal. The solution is simply

$$
\begin{aligned}
& \left|c_{1}\right|^{2}=\ldots=\left|c_{N}\right|^{2}=\frac{1}{2 N}, \quad F=\frac{\eta \gamma \varepsilon^{2}}{2^{4} \cdot 63 N} \sum_{m=1}^{N} \tilde{T}_{n m}, \\
& \tilde{T}_{n m}=T_{n m}+T_{n,-m}, \quad 1 \leq n \leq N .
\end{aligned}
$$

This result allows us to express the heat transport $H_{c}$ by convection in terms of $\left(R-R_{c}\right)$ for small values of the latter parameter. Using the approximate relationship 


$$
H_{c} \approx-\left.\varepsilon^{2} \gamma \frac{\partial \theta_{2}^{(1)}}{\partial z}\right|_{z=\frac{2}{2}}, R-R_{c}=F
$$

we find in the case of two-dimensional rolls

$$
N=1: \frac{H_{c}^{\text {rolls }}}{R-R_{c}}=7 n P /\left(\frac{326795}{2^{11} .221} \gamma+8 P^{2} \eta\right)
$$

in the case of square pattern

$$
N=2: \frac{H_{c}^{\text {squares }}}{R-R_{c}}=14 n P /\left(\frac{75.14529}{2^{11} .221} \gamma+11 P^{2} n\right)
$$

and in the case of hexagonal pattern

$$
N=3: \frac{H_{c}^{\text {hexagons }}}{R-R_{c}}=7 n P /\left(\frac{898955}{2^{10} .663} \gamma+8 P^{2} \eta\right) \text {. }
$$

It is clearly seen from (13) that, in contrast to the case discussed in [3], rolls exhibit a higher heat transport than hexagons. For $p^{2} \gg \gamma$, square cells transport more heat than rolls. The situation is reversed for $p^{2} \ll \gamma$. For $p^{2}=O(\gamma)$, we must compare (13a) and (13b). We find that square cells transport more heat than rolls, provided

$$
\gamma<15.62 P^{2} ;
$$

otherwise, rolls transport more heat than squares. Another interesting result which can be derived from (13) is that the heat transport exhibits a a non-monotonicity behavior with respect to $p$ at a given $R$. For $P \leq \gamma^{\frac{1}{2}} / 5.77, H_{c}^{\text {rolls }}$ increases with $P$ and decreases with increasing $P$ in the range $P>\gamma^{\frac{1}{2}} / 5.77$. Similar behavior is exhibited by $H_{c}^{\text {squares }}$. For $P \leq \gamma^{\frac{1}{2}} / 3.95, H_{c}^{\text {squares }}$ increases with $P$ and decreases with increasing $P$ in the range $P>\gamma^{\frac{1}{2}} / 3.95$. As the stability analysis to be discussed next indicate $H_{c}^{\text {rolls }}$ and $H_{c}^{\text {squares }}$ reach their peaks in the ranges where rolls and squares are the stable flow patterns, respectively. Hence the stable flow pattern, as expected, transports heat more 
efficiently than the unstable ones.

To distinguish the physically realizable solution among all possible steady solutions, the stability of $v, \theta$ must be investigated. The equations for the time-dependent disturbances $v, \theta$ are given by

$$
\begin{aligned}
\nabla^{4} \Delta_{2} \tilde{v}-\Delta_{2} \tilde{\theta}-\sigma \nabla^{2} \Delta_{2} \tilde{v} & =\delta \cdot(\delta \tilde{v} \cdot \nabla \delta v+\delta v \cdot \nabla \delta \tilde{v}), \\
\nabla^{2} \tilde{\theta}-R \Delta_{2} \tilde{v}-P \sigma \tilde{\theta} & =P(\delta \tilde{v} \cdot \nabla \theta+\delta v \cdot \nabla \tilde{\theta}),
\end{aligned}
$$

where we have introduced a growth rate $\sigma$ by $\partial / \partial t=\sigma$. The equations (15) are soluved by the following expansion

$$
\tilde{(\tilde{\theta}})=\sum_{n=1, m=0} \varepsilon^{n-1} \gamma^{m}\left(\begin{array}{l}
\tilde{v}_{n}^{(m)} \\
\tilde{\theta}_{n}^{(m)}
\end{array}\right), \sigma=\sum_{n, m=0} \varepsilon^{n} \gamma^{m} \sigma_{n}^{(m)} .
$$

As in [3], the investigation of the order $\varepsilon^{0} \gamma^{m}$ of (15) yields $\sigma_{0}^{(m)} \leq 0$ if $\eta=n_{c}$. Restricting the attention to the most dangerous disturbances, we assume $\sigma_{0}^{(m)}=0$. Using the representation

$$
\tilde{w}(x, y)=\sum_{n=-\infty}^{\infty} \tilde{c}_{n} \exp \left(\dot{I k}_{n} \cdot r\right)
$$

for the horizontal dependence of the general three-dimensional disturbance, we consider (15) in higher orders of $\varepsilon$. The possibility of a nonvanishing positive coefficient(s) $\sigma_{n}^{(m)}$ appears first in the order $\varepsilon^{2} \gamma^{2}$, where the solvability condition yields the following systems of equations

$$
P G \tilde{c}_{n}+\left(\frac{11 \varepsilon^{2} \gamma^{2}}{2^{6} \cdot 3^{3} \cdot 85}\right) \sum_{m=-N}^{N} \tilde{T}_{n m m} c_{m}^{*} \tilde{c}_{m} c_{n}=0,
$$

where

$$
G=\varepsilon^{2} \gamma^{2} \sigma_{2}^{(2)}+\varepsilon^{2} \gamma^{3} \sigma_{2}^{(3)}
$$

Following Schlüter et al [5], it follows that $N$ eigenvalues $G$ of the characteristic equation for the system (18) are zero. Restricting our attention to regular solutions satisfying (11) and following [3], we find that all the solutions with $N \geq 3$ are unstable. Discarding the $N$ 
eigenvalues $G=0$, the characteristic equation for (18) can be written in the form

$$
\operatorname{Det}\left|P G \delta_{n m}+\tilde{T}_{n m} c_{m}^{*} c_{n}\right|=0, n, m=1, \ldots, N
$$

For $N=1$, the root $G$ of the equation (19) is negative. For square pattern, the steady motion is stable only if

$$
\tilde{T}_{11} \geq \tilde{T}_{12}>0 \text {. }
$$

Using (10), we find that (20) holds only when the condition (14) (with the sign ( replaced by $\leq$ ) is satisfied.

So far the analysis was restricted to disturbances whose wave number vectors were coincided with those of the steady motion. We now examine the stability of the steady motion with respect to disturbances whose wave number vectors are not coincided with the basic vectors of steady motion. Defining a disturbance vector $k_{r}$, the expression for the maximum growth rate $G$ in the case $N=1$ leads to the following equation

$$
P G=\frac{11 \varepsilon^{2} \gamma^{2}}{2^{7} \cdot 3^{3} \cdot 85}\left[P^{2} n\left(1-4 \phi_{1 r}^{2}\right)+\frac{\gamma}{2^{11} \cdot 221}\left(21858 \phi_{1 \phi^{2}}^{2}-87217\right]\right]
$$

It is readily seen from (2l) that the maximum positive eigenvalue $G$ is obtained when (14) holds and $\phi_{1 r}=0$ implying that rolls are unstable to disturbances in the form of rolls oriented at a right angle to the original rolls. Hence as $\gamma \rightarrow 15.62 P^{2}$ there is a pattern transformation from rolls to squares or vice versa. The result that the preferred stable flow pattern is squares or rolls depending on whether (14) holds or not implies that the realized convection pattern transports maximum amount of heat at least in the weakly non-linear regime. This result has already been shown to hold in a layer with isothermal boundaries [1]. It is expected that in the strongly non-linear convection regime the flow field still has the tendency to maximize the heat transport. 


\section{References}

[1] F.H. Busse, "The stability of finite amplitude cellular convection and its relation to an extremum principle", J. Fluid Mech. 30 (1967), 625-249.

[2] F.H. Busse, "Non-linear properties of thermal convection", Rep. Progr. Phys. 41 (1978), 1929-1967.

[3] F.H. Busse and N. Riahi, "Nonlinear convection in a layer with nearly insulating boundaries", J. Fluid Mech. 96 (1980), 243-256.

[4] N. Riahi, "On convection with nearly insulating boundaries in a low Prandtl number fluid", 2. Angew. Math. Phys. 31 (1980), 261-266.

[5] A. Schlüter, D. Lortz and F.H. Busse, "On the stability of steady finite amplitude convection", J. Fluid Mech. 23 (1965), 129-144.

Department of Theoretical and Applied Mechanics,

College of Engineering,

University of Illinois at Urbana-Champaign, Urbana,

IIIinois 61801 , USA. 